ISSN 0103-5150

Fisioter. Mov., Curitiba, v. 25, n. 1, p. 83-92, jan./mar. 2012 Licenciado sob uma Licença Creative Commons

\title{
Relação entre independência funcional e qualidade de vida na paralisia cerebral
}

\author{
Relationship between functional independence and \\ quality of life in cerebral palsy
}

\author{
Ana Cristina Resende Camargos ${ }^{[a]}$, Tatiana Teixeira Barral de Lacerda ${ }^{[b]}$, Taise Vieira Barros ${ }^{[c]}$, \\ Gleicibele Cristina da Silva ${ }^{[\mathrm{d}]}$, Juliana Teodoro Parreiras ${ }^{[\mathrm{e}]}$, Thaís Helena de Jesus Vidal ${ }^{[\mathrm{f}]}$ \\ [a] Professora assistente da Universidade Federal dos Vales do Jequitinhonha e Mucuri (UFVJM), Diamantina, MG - Brasil, \\ e-mail: anacristina.camargos@terra.com.br \\ [b] Professora assistente da Pontifícia Universidade Católica de Minas Gerais - Câmpus Betim (PUC Betim), Betim, MG - Brasil, \\ e-mail: tatianabarral@terra.com.br \\ [c] Fisioterapeuta graduada na Pontifícia Universidade Católica de Minas Gerais - Câmpus Betim (PUC Betim), Betim, MG - \\ Brasil, e-mail: taise.vieira@gmail.com \\ [d] Fisioterapeuta graduada na Faculdade Estácio de Sá de Belo Horizonte, Belo Horizonte, MG - Brasil, e-mail: \\ gleici.andrade@yahoo.com.br \\ [e] Fisioterapeuta graduada na Faculdade Estácio de Sá de Belo Horizonte, Belo Horizonte, MG - Brasil, e-mail: \\ julyparreiras@yahoo.com.br \\ [f] Fisioterapeuta graduada na Faculdade Estácio de Sá de Belo Horizonte, Belo Horizonte, MG - Brasil, e-mail: \\ thatha.vidal@hotmail.com
}

\section{Resumo}

Introdução: A paralisia cerebral (PC) pode limitar o desempenho de atividades funcionais e a qualidade de vida desses indivíduos. Objetivos: Avaliar a relação entre independência funcional e qualidade de vida de crianças com paralisia cerebral. Materiais e métodos: A amostra foi composta por 30 crianças com diagnóstico de PC. Para mensurar a qualidade de vida foi utilizado o Questionário de Saúde da Criança (CHQ-PF50) e, para avaliar a funcionalidade, a parte I (habilidades funcionais) do Inventário de Avaliação Pediátrica de Incapacidade (Pedi). 0 teste de correlação de Spearman foi utilizado para avaliar a relação entre essas variáveis. Resultados: Foi encontrada correlação significativa entre os domínios de função física e limitação das atividades diárias graças aos aspectos emocionais e comportamentais com todas as áreas do Pedi $(\mathrm{p}<0,05)$. 0 domínio de limitação das atividades diárias graças aos aspectos físicos e a percepção 
de saúde apresentaram relação com as áreas de autocuidado e função social $(\mathrm{p}<0,05)$. A função global só apresentou relação significativa com o autocuidado, e a saúde mental apresentou relação inversa com a mobilidade $(\mathrm{p}<0,05)$. Conclusões: Nenhum domínio do CHQ-PF50 apresentou forte correlação com as áreas do Pedi, o que mostra que esses questionários avaliam constructos diferentes. Tais resultados podem auxiliar no planejamento de estratégias de avaliação e intervenção para crianças com PC.

Palavras-chave: Paralisia cerebral. Qualidade de vida. Atividades cotidianas.

\begin{abstract}
Introduction: Cerebral palsy (CP) can limit the performance of functional activities and quality of life of these individuals. Objectives: Evaluate the relationship between functional independence and quality of life of children with CP. Materials and methods: The sample was composed of 30 children with diagnosis of CP. The Child Health Questionnaire (CHQ-PF50) was used to measure the quality of life and the Part I (functional skills) of the Pediatric Evaluation of Disability Inventory (Pedi) was used to evaluate the functionality. The test of correlation Spearman was used to assess the relationship between these variables. Results: Significant correlations were found between the domains of physical function and limitation of daily activities due to emotional and behavioral aspects of all areas of the Pedi $(p<0,05)$. The domain of limitation of daily activities due to physical and perceived health were associated with areas of self care and social function $(p<0,05)$. The global function only showed a significant relationship with self-care and mental health presented an inverse relationship with the mobility $(p<0,05)$. Conclusions: No domain of CHQ-PF50 presented a strong correlation with areas of Pedi, which shows that these questionnaires assess different constructs. These results may help in planning strategies for assessment and intervention for children with $C P$.
\end{abstract}

Keywords: Cerebral palsy. Quality of life. Activities of daily living.

\section{Introdução}

A paralisia cerebral (PC) é definida como um grupo de distúrbios, caracterizados pela alteração do movimento, do tônus e da postura, que causam limitações da atividade atribuídas a distúrbios não progressivos ocorridos durante o desenvolvimento cerebral (1). Nos países desenvolvidos apresenta uma prevalência de 1,5 a 3 para cada 1.000 nascidos vivos (2). No Brasil, estima-se que a prevalência seja de 7 para cada 1.000 crianças nascidas vivas (3).

Além das desordens motoras da PC, essas crianças frequentemente apresentam distúrbios de sensação, cognição, comunicação, percepção, comportamento e presença de convulsões (1). Todas essas alterações podem limitar o desempenho de atividades funcionais e ocasionar prejuízos na realização das atividades de vida diária dessas crianças (4).

Dessa forma, crianças com PC podem apresentar limitações para realizar suas atividades de alimentação, higiene, vestuário e locomoção, além de restrição para sua participação na escola e na comunidade (5). Essas limitações e restrições podem afetar a rotina das crianças e de seus familiares e, consequentemente, a qualidade de vida desses indivíduos (5-7).

De acordo com a Classificação Internacional de Funcionalidade, Incapacidade e Saúde (CIF), proposta pela Organização Mundial da Saúde (8), a funcionalidade do indivíduo é resultante da interação entre sua condição de saúde, estruturas e funções fisiológicas, capacidade e desempenho nas atividades e participação social. Todos esses níveis podem ser influenciados por fatores pessoais e ambientais, podendo estes atuar como facilitadores ou barreiras para a realização de atividades e para a participação na sociedade (8).

Um dos instrumentos mais utilizados para mensurar a funcionalidade é o Inventário de Avaliação Pediátrica de Incapacidade (Pediatric Evaluation of Disability Inventory - Pedi) (9). De acordo com o modelo da OMS, o Pedi compreende a avaliação dos níveis de atividade e participação de crianças com incapacidades (10).

Além da importância da realização de medidas de funcionalidade, nas últimas décadas houve 
crescente interesse a respeito da qualidade de vida de crianças com incapacidades. Estudos têm demonstrado que crianças com PC apresentam escores inferiores de qualidade de vida quando comparadas à população normativa (11-13).

Segundo Lim e Zebrack (14), qualidade de vida é um constructo multidimensional, subjetivo, e está relacionado ao bem-estar físico, psicológico, social e espiritual. Em crianças, o termo qualidade de vida não inclui somente conceitos da doença, estado funcional, saúde mental e conforto, mas o impacto de funcionamento familiar também deve ser levado em consideração (7). Além disso, alguns estudos descrevem que muitas definições de qualidade de vida se baseiam fortemente na funcionalidade (15).

A qualidade de vida de crianças com PC apresenta muita dificuldade em ser medida, graças à dificuldade de interpretação e de expressão nas crianças com algum comprometimento cognitivo ou de fala. Dentre os instrumentos disponíveis na literatura, o Questionário de Saúde das Crianças Relatório dos Pais 50 (Child Health Questionnaire Parent Form 50 - CHQ-PF50) parece ser uma ferramenta útil, uma vez que permite avaliar esse aspecto sob a perspectiva dos pais (16). Esse instrumento é considerado um dos mais utilizados na literatura para avaliar a qualidade de vida das crianças com PC $(12,13,17)$.

Independência funcional e qualidade de vida podem ser citadas como as principais metas da equipe de reabilitação responsável pelo tratamento de crianças com PC. O conhecimento da relação entre esses fatores pode aprimorar o desenvolvimento de estratégias de intervenção para essa população. Dessa forma, o objetivo desse estudo foi avaliar a relação entre a independência funcional e a qualidade de vida de crianças com PC.

\section{Materiais e métodos}

Foi realizado um estudo transversal no período de maio a outubro de 2008, que foi aprovado pelo Comitê de Ética e Pesquisa da Pontifícia Universidade Católica de Minas Gerais (PUC Minas) (CAAE 0164.0.213.000-07).

Foram entrevistados os pais ou cuidadores de 30 crianças com diagnóstico clínico de PC que realizavam tratamento fisioterapêutico no Centro Clínico de Fisioterapia da PUC Betim e na Associação de
Pais e Amigos de Crianças Excepcionais em Betim (Apae Betim).

Para participar do estudo era necessário à criança preencher os seguintes critérios de inclusão: ter pais ou cuidadores alfabetizados, apresentar diagnóstico clínico de PC, ter idade igual ou superior a cinco anos, estar em tratamento fisioterapêutico e os responsáveis assinarem o Termo de Consentimento Livre e Esclarecido (TCLE), concordando em participar do estudo. Foram excluídas do estudo as crianças que possuíam diagnóstico associado de alguma doença ou síndrome neuromuscular além da PC.

Para avaliar a qualidade de vida das crianças com PC foi utilizado o Questionário de Saúde da Criança Relatório dos Pais 50 (CHQ-PF50) (16). Esse questionário corresponde a um instrumento genérico de qualidade de vida relacionado à saúde, que foi desenvolvido pelo Child Health Assessment Project, no período de 1990 a 1996 (18). Tal questionário foi traduzido, validado e adaptado culturalmente para a população brasileira por Machado et al. (19), e suas propriedades psicométricas foram consideradas adequadas para crianças e adolescentes brasileiros com PC por Morales et al. (13).

Esse instrumento destina-se a crianças com idade entre 5 e 19 anos e avalia 14 domínios de saúde sob a perspectiva dos pais ou responsáveis (18). Cada domínio tem uma pontuação de 0 a 100; uma maior pontuação indica melhor estado de saúde, grau de satisfação e bem-estar. 0 questionário compreende os domínios de saúde global, função física, limitação das atividades diárias em razão dos aspectos emocionais e comportamentais, limitação das atividades diárias pelos aspectos físicos, dor corporal, comportamento, saúde mental, autoestima, percepção de saúde, alteração de saúde, impacto emocional nos pais, impacto no tempo dos pais, atividade familiar e coesão familiar (16).

Para avaliar a independência funcional das crianças com PC foi utilizado o Inventário de Avaliação Pediátrica de Incapacidade (Pedi). Esse instrumento de avaliação infantil informa sobre o desempenho funcional de crianças entre 6 meses a 7 anos e 6 meses de idade, mas pode ser utilizado com crianças com idade superior desde que o desempenho funcional seja compatível com o de crianças dessa faixa etária. Tal inventário foi traduzido para o português e adaptado culturalmente para contemplar as especificidades socioculturais brasileiras (9). 
O Pedi consiste de um questionário estruturado que pode ser administrado por meio de entrevista com os pais ou cuidadores das crianças. Ele compreende três partes: I - habilidades funcionais (197 itens); II - assistência do cuidador (20 itens); e III modificações do ambiente (20 itens). A parte I informa sobre a capacidade da criança em realizar atividades e tarefas de seu cotidiano. A parte II informa sobre a quantidade de assistência que o cuidador deve fornecer para a criança realizar as atividades e tarefas do cotidiano. A parte III informa sobre a quantidade de modificações do ambiente utilizadas pela criança. Cada parte é ainda subdividida em três áreas de função: autocuidado, mobilidade e função social (9). Nesse estudo foi utilizada somente a parte I, com suas três áreas de função.

Após a assinatura do TCLE foi realizada uma coleta de dados a respeito de idade, gênero, diagnóstico topográfico e nível de comprometimento motor para caracterização da amostra.

O nível de comprometimento motor foi avaliado por meio do Sistema de Classificação da Função Motora Grossa (Gross Motor Function Classification System - GMFCS), que é baseado no movimento iniciado voluntariamente, enfatizando particularmente o sentar e o andar. Ele é dividido em cinco níveis, no qual o nível I é o mais leve e o nível $V$ o mais grave (20).

Em local reservado e tranquilo foram aplicados os instrumentos Pedi e CHQ-PF50. Primeiramente, o cuidador, que poderia ou não ser um dos pais, era questionado por um avaliador único e treinado sobre os itens contidos no Pedi. Em um intervalo máximo de 15 dias o cuidador era convidado a responder o CHQ-PF50, sem a interferência do avaliador, entretanto sob sua supervisão.

Todas as análises foram realizadas utilizando o programa SPSS, versão 13.0 para Windows. Primeiramente, foi realizada estatística descritiva para caracterização da amostra e depois foi utilizado o teste de correlação de Spearman para avaliar a relação de cada domínio da qualidade de vida do CHQ-PF50 com cada área do Pedi, sendo considerado significativo com o valor de $\mathrm{p}<0,05$. A força de associação entre as variáveis foi considerada de acordo com Portney e Watkins (21), em que valores de correlação entre 0.00 e 0.25 representam pouca ou nenhuma associação entre as variáveis; entre 0.25 a 0.50 fraca associação; entre 0.50 e 0.75 moderada associação; e acima de 0.75 forte associação.

\section{Resultados}

Participaram deste estudo 30 crianças, com média de idade de 7,67 anos (DP $=2,28$ ). Nove crianças eram do gênero feminino e vinte e uma do gênero masculino.

Quanto ao tipo de PC espástica, seis crianças apresentavam quadriplegia; doze, diplegia; e seis, hemiplegia. Além disso, uma criança apresentava ataxia; três, discinesia; e duas apresentavam tipo misto.

Em relação à classificação no GMFCS, nove crianças se encontravam no nível I, seis no nível II, duas no nível III, duas no nível IV e onze no nível V.

Os resultados dos escores de cada domínio do CHQ-PF50 estão descritos na Tabela 1.

A Tabela 2 demonstra os domínios do CHQ-PF50 que apresentaram correlação com as áreas da Pedi.

Foi observada correlação significativa moderada entre o domínio de função física e as áreas de autocuidado e mobilidade, entre o domínio limitação das atividades diárias pelos aspectos físicos e a área de função social e entre o domínio limitação das atividades diárias em razão dos aspectos emocionais e comportamentais com a área de função social.

Foi encontrada correlação significativa fraca entre o domínio de saúde global e a área autocuidado, entre o domínio percepção de saúde e as áreas de autocuidado e função social, entre o domínio de função física e a área de função social, entre o domínio limitação das atividades diárias pelos aspectos físicos e a área de autocuidado e entre o domínio limitação das atividades diárias em razão dos aspectos emocionais e comportamentais com as áreas de autocuidado e mobilidade. 0 domínio de saúde mental apresentou correlação negativa significativa e fraca com a área de mobilidade.

Os domínios de dor corporal, comportamento, autoestima, alteração de saúde, impacto emocional nos pais, impacto no tempo dos pais, atividade familiar e coesão familiar não apresentaram correlação significativa com nenhuma das áreas do Pedi.

\section{Discussão}

A literatura aponta que existe confusão a respeito dos termos bem-estar, saúde e funcionalidade nos instrumentos de qualidade de vida $(22,23)$. De acordo com Shelly et al. (23), instrumentos de qualidade de vida utilizam medidas tanto de bem-estar 
Tabela 1 - Escores obtidos nos domínios do Questionário de Saúde da Criança (CHQ-PF50)

\begin{tabular}{llc}
\hline Domínios & Média & Desvio-padrão \\
\hline Saúde global & 71,33 & 25,12 \\
Função física & 51,82 & 31,43 \\
Limitação das atividades diárias em razão dos aspectos emocionais e comportamentais & 54,90 & 29,68 \\
Limitação das atividades diárias pelos aspectos físicos & 50,30 & 36,94 \\
Dor corporal & 75,33 & 27,00 \\
Comportamento & 65,48 & 18,62 \\
Saúde mental & 67,17 & 17,20 \\
Autoestima & 83,31 & 17,71 \\
Percepção de saúde & 59,83 & 20,70 \\
Alteração de saúde & 84,17 & 22,25 \\
Impacto emocional nos pais & 58,59 & 25,57 \\
Impacto no tempo dos pais & 67,08 & 27,85 \\
Atividade familiar & 75,11 & 19,03 \\
Coesão familiar & 72,50 & 25,45 \\
\hline
\end{tabular}

Fonte: Dados da pesquisa.

Tabela 2 - Relação entre os domínios do Questionário de Saúde da Criança (CHQ-PF50) e o Inventário de Avaliação Pediátrica de Incapacidade (Pedi)

(Continua)

\begin{tabular}{|c|c|c|c|c|}
\hline \multirow[b]{2}{*}{ Domínios do CHQ-PF50 } & & \multicolumn{3}{|c|}{ Áreas do Pedi } \\
\hline & & Autocuidado & Mobilidade & Função social \\
\hline \multirow{2}{*}{ Saúde global } & r & 0,43 & 0,26 & 0,20 \\
\hline & $\mathrm{p}$ & $0,018^{*}$ & 0,17 & 0,29 \\
\hline \multirow{2}{*}{ Função física } & r & 0,65 & 0,63 & 0,48 \\
\hline & $p$ & $0,0001^{*}$ & $0,0001^{*}$ & $0,008^{*}$ \\
\hline \multirow{2}{*}{$\begin{array}{l}\text { Limitação das atividades diárias em razão dos aspectos } \\
\text { emocionais e comportamentais }\end{array}$} & r & 0,49 & 0,39 & 0,65 \\
\hline & $p$ & $0,006^{*}$ & $0,03^{*}$ & $0,0001^{*}$ \\
\hline \multirow{2}{*}{ Limitação das atividades diárias pelos aspectos físicos } & r & 0,39 & 0,21 & 0,55 \\
\hline & $p$ & $0,03^{*}$ & 0,26 & $0,002^{*}$ \\
\hline \multirow{2}{*}{ Dor corporal } & r & $-0,18$ & $-0,24$ & $-0,19$ \\
\hline & $p$ & 0,34 & 0,20 & 0,31 \\
\hline \multirow{2}{*}{ Comportamento } & $r$ & $-0,20$ & $-0,26$ & $-0,01$ \\
\hline & $p$ & 0,29 & 0,17 & 0,95 \\
\hline
\end{tabular}


Tabela 2 - Relação entre os domínios do Questionário de Saúde da Criança (CHQ-PF50) e o Inventário de Avaliação Pediátrica de Incapacidade (Pedi)

(Conclusão)

\begin{tabular}{|c|c|c|c|c|}
\hline \multirow[b]{2}{*}{ Domínios do CHQ-PF50 } & & \multicolumn{3}{|c|}{ Áreas do Pedi } \\
\hline & & Autocuidado & Mobilidade & Função social \\
\hline \multirow{2}{*}{ Saúde mental } & $r$ & $-0,33$ & $-0,44$ & 0,08 \\
\hline & $p$ & 0,08 & $0,01^{*}$ & 0,67 \\
\hline \multirow{2}{*}{ Autoestima } & $r$ & 0,16 & $-0,09$ & 0,20 \\
\hline & $\mathrm{p}$ & 0,39 & 0,64 & 0,29 \\
\hline \multirow{2}{*}{ Percepção de saúde } & r & 0,48 & 0,32 & 0,49 \\
\hline & $p$ & $0,007^{*}$ & 0,08 & $0,006^{*}$ \\
\hline \multirow{2}{*}{ Alteração de saúde } & $r$ & 0,10 & 0,16 & $-0,10$ \\
\hline & $p$ & 0,61 & 0,39 & 0,60 \\
\hline \multirow{2}{*}{ Impacto emocional nos pais } & $r$ & $-0,18$ & $-0,32$ & $-0,10$ \\
\hline & $\mathrm{p}$ & 0,34 & 0,08 & 0,61 \\
\hline \multirow{2}{*}{ Impacto no tempo dos pais } & r & 0,21 & 0,12 & 0,31 \\
\hline & $p$ & 0,26 & 0,53 & 0,09 \\
\hline \multirow{2}{*}{ Atividade familiar } & r & 0,18 & $-0,06$ & 0,35 \\
\hline & $p$ & 0,34 & 0,75 & 0,06 \\
\hline \multirow{2}{*}{ Coesão familiar } & $r$ & 0,10 & 0,15 & 0,32 \\
\hline & $p$ & 0,61 & 0,43 & 0,08 \\
\hline
\end{tabular}

Legenda: * $=$ correlação significativa entre as variáveis; $p<0,05$.

Fonte: Dados da pesquisa.

quanto de funcionalidade. Esses autores ainda relatam que muitas definições equiparam qualidade de vida com funcionalidade ou se baseiam fortemente em funcionalidade. Dessa forma, é importante que o profissional da área da saúde conheça as definições operacionais do instrumento utilizado. Apesar do CHQ-PF50 avaliar bem-estar e funcionalidade, esse instrumento não apresentou forte correlação com nenhuma das áreas do Pedi, o que indica que esses instrumentos avaliam constructos diferentes.

O domínio de função física do CHQ-PF50 apresentou moderada associação com as áreas de autocuidado e mobilidade da Pedi e fraca associação com a área de função social do Pedi. Esses achados foram semelhantes ao estudo de McCarthy et al. (24), que também encontraram essa relação entre as variáveis. De acordo com Mancini et al. (25), as deficiências neuromotoras apresentadas pelas crianças com PC podem resultar em limitações no desempenho de atividades e tarefas do cotidiano. Esse domínio do CHQ-PF50 refere-se às atividades físicas realizadas pelas crianças durante o dia, como, por exemplo, correr, jogar futebol, andar de bicicleta ou patins, subir um lance de escadas ou alimentar-se, vestir-se e ir ao banheiro. Muitos desses itens são semelhantes aos utilizados nas áreas de autocuidado e mobilidade do Pedi. Cabe ressaltar que no estudo de Wake et al. (11), que também utilizou o CHQ, a função física foi o fator que representou o maior impacto na saúde da criança com PC de acordo com a percepção dos pais.

Ainda em relação à parte física do instrumento, pode-se perceber que a limitação das atividades diárias pelos aspectos físicos apresentou moderada associação com a função social e fraca associação com o autocuidado. Esse domínio de qualidade de 
vida refere-se às limitações das crianças realizarem trabalhos escolares em razão de problemas de saúde física. Para Lepage et al. (6), quanto maior o comprometimento da criança com PC, mais restrições ela encontra no desempenho de atividades sociais, em decorrência não só das dificuldades de se engajar em atividades comuns às de outras crianças, como também pela dificuldade de executá-las com sucesso e integrar-se ao grupo. De acordo com Majnemer et al. (17), limitações motoras, de comunicação, socialização e atividades de vida diária estão mais relacionadas com o bem-estar físico do que com o bem-estar psicossocial.

Dentro dos aspectos psicossociais do CHQ-PF50, o domínio de limitação das atividades diárias em razão dos aspectos emocionais e comportamentais apresentou moderada associação com a área de função social e fraca associação com as áreas de autocuidado e mobilidade. Esse domínio de qualidade de vida engloba as limitações em realizar trabalhos escolares por consequência de problemas de comportamento das crianças. De acordo com Majnemer et al. (17), problemas comportamentais normalmente limitam a função social de crianças com PC. Esses autores ainda relatam que as dificuldades comportamentais e de socialização estão relacionadas com a diminuição do bem-estar psicossocial das crianças, uma vez que elas não têm capacidade de se adaptar às demandas do dia a dia. Além disso, Morales (26) descreve que as limitações das atividades em razão dos aspectos emocionais e comportamentais estão relacionadas com maior gravidade do comprometimento motor.

Ainda relacionado aos aspectos psicossociais, a saúde mental apresentou associação inversa e fraca com a área de mobilidade. Esse domínio reflete o estado de humor das crianças e, de acordo com o resultado do presente estudo, quanto melhor o estado de humor da criança, pior sua mobilidade. Esse resultado foi inesperado, uma vez que o esperado era que a relação fosse direta, e não inversa. Os estudos de McCarthy et al. (24) e Liptak et al. (27) também apontaram inadequação desse domínio para crianças com PC. Morales (26) sugere comparar essa escala com instrumentos mais específicos que avaliem o constructo desse domínio, uma vez que também identificou aspectos divergentes nessa escala.

Foi encontrada também fraca associação entre o domínio saúde global e as habilidades funcionais de autocuidado. Dessa forma, pode-se sugerir que o estado de saúde da criança com PC influencia, mesmo que de forma fraca, sua habilidade de realizar habilidades de autocuidado como alimentação, higiene e vestuário. Além disso, houve também fraca associação entre a percepção de saúde e as áreas de autocuidado e função social. Esse domínio do CHQ-PF50 avalia a percepção dos pais sobre o estado de saúde no passado, presente e futuro da criança, bem com sua suscetibilidade para adoecer. Isso significa que crianças que adoecem com facilidade apresentam dificuldade em realizar atividades diárias e sociais. Sabe-se que as crianças com PC apresentam vários distúrbios associados que podem comprometer ainda mais o seu quadro clínico (28). De acordo com Pruitt e Tsai (29), a presença desses distúrbios pode alterar a saúde e a qualidade de vida dessas crianças. Além disso, pode interferir na execução das atividades de vida diária e restringir participação social de crianças com PC $(28,30)$.

Os domínios de dor corporal, comportamento, autoestima, alteração de saúde, impacto emocional nos pais, impacto no tempo dos pais, atividade familiar e coesão familiar não apresentaram correlação significativa com as áreas do Pedi. Apesar de não haver relação entre essas variáveis neste estudo, esses domínios também são discutidos na literatura. De acordo com Dickinson et al. (31), a dor corporal está associada com piores escores de qualidade de vida. Além disso, a dor corporal e a autoestima podem diminuir com a idade em crianças com PC (12). Majnemer et al. (17) descrevem que as alterações de comportamento estão fortemente associadas à redução do bem-estar psicossocial. Esses autores ainda dizem que baixos escores de qualidade de vida estão associados aos domínios de impacto emocional nos pais, impacto no tempo dos pais e atividade familiar, mas não estão relacionados à coesão familiar.

O domínio alteração de saúde apresentou escore superior à população normativa do estudo de adaptação cultural brasileira realizado por Machado et al. (19), diferente dos outros domínios do CHQPF50, que apresentaram escores médios inferiores a essa população. Esses achados também foram encontrados no estudo de Morales (26). A alteração de saúde é avaliada pela percepção dos pais ou responsáveis em relação à saúde de seu/sua filho(a) quando comparada ao ano anterior. Assim, maiores escores indicam que a saúde da criança está melhor que há um ano. Como geralmente as crianças saudáveis não apresentam grandes mudanças no seu estado de saúde, a maior parte apresentou o escore 
médio, que representa que a saúde da criança está da mesma forma que no ano anterior. Entretanto, crianças com PC podem apresentar vários distúrbios associados, como epilepsia, alterações cardiorrespiratórias, ortopédicas, gastrointestinais, dentre outras, que podem alterar a saúde, a qualidade e a expectativa de vida dessas crianças (29). A melhora percebida pelos pais em relação à saúde de um ano atrás pode ser inferida pelo fato dessas crianças estarem em tratamento interdisciplinar em locais de reabilitação, o que pode minimizar as complicações de saúde. De acordo com Morales (26), a possibilidade de apresentar melhora na saúde no período de um ano é encarada com otimismo pelos familiares.

Deve ser salientado que este estudo foi desenvolvido a partir de dados coletados mediante a percepção subjetiva dos pais. Apesar de os questionários apresentarem adequada aplicabilidade, fatores pessoais, emocionais e o nível do entendimento dos pais podem interferir de forma indireta sobre os dados. Para Faleiros e Machado (32), a opinião dos pais é relevante e importante quando presentes e atuantes são capazes de estimular o bem-estar global das crianças. Entretanto, a PC pode promover profundos efeitos sobre o dia a dia das crianças e suas famílias (12). Dessa forma, o estresse dos pais e a dificuldade com as estratégias de enfretamento podem afetar negativamente os escores de qualidade de vida (17). De acordo com o estudo de Faleiros e Machado (32), diferenças têm sido observadas quando os questionários de qualidade de vida são respondidos pelas crianças e pelos pais. Entretanto, em razão das dificuldades cognitivas apresentadas por crianças com esse quadro clínico, a obtenção de respostas por elas é muito complicada (33).

\section{Conclusão}

Foi observada relação entre os domínios de função física e limitação das atividades diárias em razão dos aspectos emocionais e comportamentais com todas as áreas do Pedi. 0 domínio de limitação das atividades diárias pelos aspectos físicos e a percepção de saúde apresentaram relação com as áreas de autocuidado e função social. A função global só apresentou relação significativa com o autocuidado, e a saúde mental apresentou relação inversa com a mobilidade. Os demais domínios de qualidade de vida não apresentaram relação significativa com nenhuma área do Pedi.
Entretanto, não foi observada forte relação entre nenhum domínio de qualidade de vida e as áreas do Pedi, o que indica que esses instrumentos avaliam constructos diferentes. Dessa forma, o CHQ-PF50 deve ser utilizado com o objetivo de avaliar a qualidade de vida genérica da criança e o Pedi com objetivo de avaliar aspectos específicos da funcionalidade. É importante apontar que os profissionais da área de reabilitação devem avaliar e tratar seus pacientes com enfoque tanto na funcionalidade quanto na qualidade de vida.

Em relação a toda literatura consultada, este é primeiro estudo que investigou a relação entre o CHQ-PF50 e o Pedi e, portanto, apresenta informações importantes dentro dos aspectos de funcionalidade e qualidade de vida. Dessa forma, são necessários novos estudos que avaliem o impacto da funcionalidade na qualidade de vida. Tais resultados podem auxiliar no planejamento de estratégias de avaliação e intervenção para crianças com PC.

\section{Agradecimentos}

Ao auxílio financeiro do FIP PUC Minas.

\section{Referências}

1. Bax M, Goldstein M, Rosenbaum P, Leviton A, Paneth N, Dan B, et al. Proposed definition and classification of cerebral palsy. Dev Med Child Neurol. 2005; 47(8):571-6.

2. Surveillance of Cerebral Palsy in Europe. Surveillance of cerebral palsy in Europe: a collaboration of cerebral palsy surveys and registers. Surveillance of cerebral palsy in Europe (SCPE). Dev Med Child Neurol. 2000;42(12):816-24.

3. Piovesana AMSG. Encefalopatia crônica (paralisia cerebral): etiologia, classificação, tratamento clínico. In: Fonseca LF, Pianetti G, Xavier CC, editores. Compêndio de neurologia infantil. Rio de Janeiro: MEDSI; 2002. p. 825-38.

4. Charles JR, Wolf SL, Schneider JA, Gordon AM. Efficacy of a child-friendly form of constraint-induced movement therapy in hemiplegic cerebral palsy: a randomized control trial. Dev Med Child Neurol. 2006;48(8):635-42. 
5. Gordon A, Connelly A, Neville B, Vargha-Khadem F, Jessop N, Murphy T, et al. Modified constraint-induced movement therapy after childhood stroke. Dev Med Child Neurol. 2007;49(1):23-7.

6. Lepage C, Noreau L, Bernard PM. Association between characteristics of locomotion an accomplishment of life habits in children with cerebral palsy. Phys Ther. 1998;78(5):458-69.

7. Vargus-Adams J. Health-related quality of life in childhood cerebral palsy. Arch Phys Med Rehabil. 2005; 86(5):940-5.

8. Organização Mundial da Saúde (OMS), Organização Panamericana de Saúde (OPAS). CIF - Classificação Internacional de Funcionalidade, Incapacidade e Saúde. São Paulo: USP; 2003.

9. Mancini MC. Inventário de avaliação pediátrica de incapacidade (PEDI): manual da versão brasileira adaptada. Belo Horizonte: Ed. UFMG; 2005.

10. Harvey A, Robin J, Morris ME, Graham HK, Baker R. A systematic review of measures of activity limitation for children with cerebral palsy. Dev Med Child Neurol. 2008;50(3):190-8.

11. Wake M, Salmon L, Reddihough D. Health status of Australian children with mild to severe cerebral palsy: cross-sectional survey using the Child Health Questionnaire. Dev Med Child Neurol. 2003; 45(3):194-9.

12. Vargus-Adams J. Longitudinal use of the child health questionnaire in childhood cerebral palsy. Dev Med Child Neurol. 2006;48(5):343-7.

13. Morales NM, Silva CH, Frontarolli AC, Araújo RR, Rangel VO, Pinto RM, et al. Psychometric properties of the initial Brazilian version of the CHQ-PF50 applied to the caregivers of children and adolescents with cerebral palsy. Qual Life Res. 2006;16(3):437-44.

14. Lim JW, Zebrack B. Caring for family members with chronic physical illness: a critical review of caregiver literature. Health Qual Life Outcomes. 2004;2:50.

15. Davis E, Waters E, Mackinnon A, Reddihough D, Graham HK, Mehmet-Radji O, et al. Paediatric quality of life instruments: a review of the impact of the conceptual framework on outcomes. Dev Med Child Neurol. 2006;48(4):311-8.
16. HealthActCHQ. The CHQ scoring and interpretation manual. Boston, MA: HealthActCHQ; 2008.

17. Majnemer A, Shevell M, Rosenbaum P, Law M, Poulin C. Determinants of life quality in school-age children with cerebral palsy. J Pediatr. 2007;151(5):470-5.

18. Landgraf JM, Abetz I, Ware JE. The CHQ: a user's manual. Boston (MA): The Health Institute, New England Medical Center; 1999.

19. Machado CS, Ruperto N, Silva CH, Ferriani VP, Roscoe I, Campos LM, et al. The Brazilian version of the Childhood Health Assessment Questionnaire (CHAQ) and the Child Health Questionnaire (CHQ). Clin Exp Rheumatol. 2001;19(4 suppl 23):25-9.

20. Palisano R, Rosenbaum P, Walter S, Russell D, Wood E, Galuppi B. Development and reliability of a system to classify gross motor function in children with cerebral palsy. Dev Med Child Neurol. 1997; 39(4):214-23.

21. Portney LG, Watkins MP. Foundations of clinical research: applications to practice. New Jersey: Prentice Hall Health; 2000.

22. Rosenbaum PL, Livingston MH, Palisano RJ, Galuppi BE, Russell DJ. Quality of life and health-related quality of life of adolescents with cerebral palsy. Dev Med Child Neurol. 2007;49(7):516-21.

23. Shelly A, Davis E, Waters E, Mackinnon A, Reddihough D, Boyd R, et al. The relationship between quality of life and functioning for children with cerebral palsy. Dev Med Child Neurol. 2008;50(3):199-203.

24. McCarthy ML, Silberstein CE, Atkins EA, Harryman SE, Sponseller PD, Hadley-Miller NA. Comparing reliability and validity of pediatric instruments for measuring health and well-being of children with spastic cerebral palsy. Dev Med Child Neurol. 2002; 44(7):468-76.

25. Mancini MC, Fiúza PM, Rebelo JM, Magalhães LC, Coelho ZAC, Paixão ML, et al. Comparação do desempenho de atividades funcionais em crianças com desenvolvimento normal e crianças com paralisia cerebral. Arq neuropsiquiatr. 2002;60(2B):446-52.

26. Morales NMO. Avaliação transversal da qualidade de vida em crianças e adolescentes com paralisia cerebral por meio de um instrumento genérico (CHQPF50) [dissertação]. Uberlândia: Ed. UFU; 2005. 
27. Liptak GS, O’Donnell M, Conaway M, Chumlea WC, Wolrey G, Henderson RC, et al. Health status of children with moderate to severe cerebral palsy. Dev Med Child Neurol. 2001;43(6):364-70.

28. Ostensjo S, Carlberg EB, Vollestad NK. Motor impairments in young children with cerebral palsy: relationship to gross motor function and everyday activities. Dev Med Child Neurol. 2004;46(9):580-9.

29. Pruitt DW, Tsai T. Common medical comorbidities associated with cerebral palsy. Phys Med Rehabil Clin N Am. 2009;20(3):453-67.

30. Beckung E, Hagberg G. Neuroimpairments, activity limitations, and participation restrictions in children with cerebral palsy. Dev Med Child Neurol. 2002; 44(5):309-16.
31. Dickinson HO, Parkinson KN, Ravens-Sieberer U, Schirripa G, Thyen U, Arnaud C, et al. Self-reported quality of life of 8-12-year-old children with cerebral palsy: a cross-sectional European study. Lancet. 2007;369(9580):2171-8.

32. Faleiros FTV, Machado NC. Avaliação da qualidade de vida relacionada à saúde em crianças com distúrbios funcionais da defecação. J Pediatr. 2006;82(6):421-5.

33. Bjornson KF, McLaughlin JF. The measurement of health-related quality of life (HRQL) in children with cerebral palsy. Eur J Neurol. 2001;8(suppl 5):183-93.

Recebido: 05/01/2011

Received: 01/05/2011

Aprovado: 23/05/2011

Approved: 05/23/2011 